\title{
Tribological Characteristics of Ultra-thin DLC Films Prepared by Filtered Cathodic Vacuum Arc
}

\author{
Nobuto Yasui, Hiroshi Inaba, Nobuyoshi Kasahara ${ }^{1}$, Hiroya Murakami ${ }^{1}$ and Naoto Ohtake ${ }^{2}$ \\ Central Research Laboratory, Hitachi, Ltd., 292 Yoshida-cho, Totsuka-ku, Yokohama, 244-0817, Japan \\ Fax: 81-45-860-2433, e-mail: nobuto.yasui.cy@hitachi.com \\ ${ }^{1}$ Department of Mechanical Sciences and Engineering, Tokyo Institute of Technology, \\ 2-12-1, O-Okayama, Meguro-ku, Tokyo, 152-8552, Japan \\ ${ }^{2}$ Graduate School of Engineering, Nagoya University, Furo-cho, Chikusa-ku, Nagoya, 464-8603, Japan
}

Recently, to meet the demands of emerging industries, such as magnetic storage devices and micro electro mechanical systems (MEMS), diamond-like carbon (DLC) coatings have been required to be ultra-thin and sufficiently durable. The present study employed frictional force microscopy (FFM) to measure the coefficient of friction (COF) of ultra-thin DLC films, and thus, characterize their mechanical durability. FFM revealed that the COF of DLC films prepared by filtered cathodic vacuum arc (FCVA) increased with decreasing thickness. To explain the above tribological behavior, several mechanisms were discussed. The indications are that an increase in contact area plays an important role in the increase of COF of ultra-thin DLC films.

Key words: DLC, AFM, FFM, Filtered cathodic vacuum arc, ultra-thin film

\section{INTRODUCTION}

DLC films have attracted an increasing attention in a variety of industries owing to their excellent mechanical and chemical properties. To meet the demands of the emerging industries, such as micro electro mechanical systems (MEMS) and magnetic storage devices, which require an ultra-thin durable overcoat, a considerable number of research studies have been devoted to advancing the technology for thin DLC film fabrication [1].

Cathodic arc discharge has been one of the most commonly used methods of fabricating thin durable DLC coatings owing to the particularly high $s p^{3}$ ratio of the resultant DLC films. However, it has been reported that, even with cathodic arc discharge, one cannot sustain high durability at thicknesses below $10 \mathrm{~nm}$. Sundararajan et al. showed that hard carbon overcoats became less wear-resistant below a thickness of $5 \mathrm{~nm}$ using frictional force microscopy (FFM) under loads ranging from 10 to $80 \mu \mathrm{N}$ [2]. Ma et al. demonstrated that plastic deformation started at lower loads for thinner films by using a surface force microscope under loads between 10 to $1200 \mu \mathrm{N}$ [3]. The mechanism of this degradation was primarily attributed to a fracture of substrate prior to that of DLC films. However, as was shown by Liu et al, an increase in COF of ultra-thin DLC films was observed at a load under $40 \mathrm{nN}$, without a significant fracture of a film and substrate [4]. This result indicates that the tribological properties of ultrathin DLC films degrade even without the fracture of substrate since high COF leads to low durability.

The present study aims to verify the relationship between the increase in COF and the various properties that change with film thickness, such as the $s p^{3}$ ratio [5], surface roughness, and thickness itself, under a normal load, which does not cause explicit plastic deformation on the surface, and finally identify the critical parameter that accounts for nano-tribological behavior of ultrathin DLC films.

\section{EXPERIMENTAL}

ta-C films were prepared by FCVA. The details of FCVA system has been described elsewhere [6]. In FCVA technique, the depositing carbon ions are generated in the arc discharge between a graphite cathode and anode. The carbon ions in the arc discharge have hyperthermal energies (several tens of $\mathrm{eV}$ ), which leads to the formation of hard carbon films with high $s p^{3}$ tatio. In the present study, an FCVA system made by Nanofilm Technology International (NTI) was used. The arc current was $30 \mathrm{~A}$, and the base pressure was $8 \times 10^{-5}$ $\mathrm{Pa}$. Working gas was not introduced during deposition.

a-C films were prepared by radio frequency (RF) magnetron sputtering. The graphite target was sputtered by Ar plasma at an RF power of $200 \mathrm{~W}$, an Ar mass flow rate of $50 \mathrm{sccm}$, and a pressure during the sputtering of $0.6 \mathrm{~Pa}$. In the case of sputtering, the depositing atoms have an energy of several $\mathrm{eV}$, i.e., lower than that in FCVA [7], which leads to lower $s p^{3}$ bonding ratio in the film [5].

The DLC films studied were prepared on Si $(100)$ substrates at various thicknesses between $1 \mathrm{~nm}$ and 50 $\mathrm{nm}$. The film thicknesses of samples were measured by spectroscopic ellipsometry and X-ray fluorescence (XRF). The surface morphology of the films was characterized by atomic force microscopy (AFM), and their frictional behavior was evaluated by FFM. A $\mathrm{Si}_{3} \mathrm{~N}_{4}$ cantilever with a tip curvature radius of approximately $20 \mathrm{~nm}$ was selected for both AFM and FFM measurement. Young's modulus of $\mathrm{Si}_{3} \mathrm{~N}_{4}$ is $285 \mathrm{GPa}$ [8]. The spring constants of the cantilever are $0.75 \mathrm{~N} / \mathrm{m}$ for normal bending and $483.3 \mathrm{~N} / \mathrm{m}$ for torsion. Surface morphologies were obtained in the contact mode over a scan area of $1 \times 1 \mu^{2}$. The procedure of FFM is 


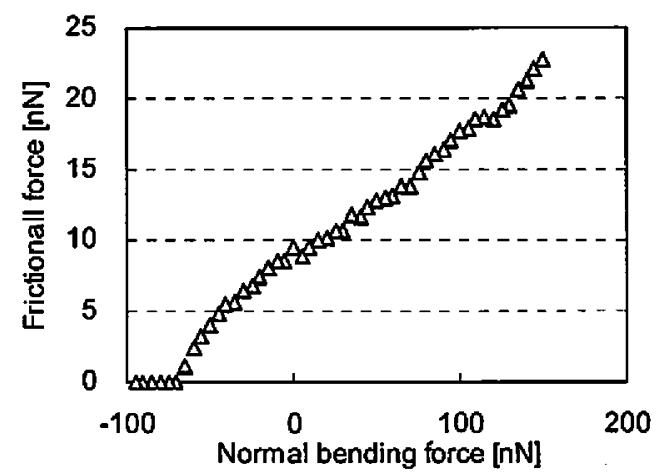

Fig. 1 A typical frictional force curve measured by FFM.

basically the same as that generally used in FFM studies $[9,10]$. Once the cantilever makes a contact with the sample surface, a load of $150 \mathrm{nN}$ is applied on the cantilever along the normal to the surface. This force is then gradually unloaded. During unloading, the frictional force is measured by observing the torsion angle of the cantilever which is repeatedly scanned back and forth. The tip of the cantilever slides over a distance of $100 \mathrm{~nm}$ at a velocity of $600 \mathrm{~nm} / \mathrm{s}$. COF was calculated from the data obtained at a normal load of less than 150 $\mathrm{nN}$ under atmospheric pressure. The humidity was $55 \pm 5 \%$. While the measurement of COF is an essential part of this study, FFM may involve two types of errors. First, the result varies from cantilever to cantilever as it is dependent on the cantilever's torsion stiffness, which is difficult to measure precisely. Second, the contact between a cantilever and sample surface is influenced by the shape of the cantilever tip and humidity, which vary with time over the course of a series of measurements. To prevent these errors from influencing the COF measurement results, the following procedure was applied. First, a series of measurements were conducted using the same cantilever so as to eliminate the error due to the stiffness variation among the supplied cantilevers. Second, to address the temporal deviation of the tip shape and humidity, we examined the reproducibility of the COF results of a 50-nm-thick ta-C film over the course of a series of measurements. The results are presented in Section 3. No significant change was observed, which indicated the reproducibility of the COF measurements under the above condition.

\section{RESULTS ANS DISCUSSION}

Figure 1 shows a typical curve obtained through FFM measurement of a 50 -nm-thick ta-C film. The zero on the normal bending force indicates an approximate point where the tip snaps into the surface during the loading, which might be due to the influence of condensed films or long range attractive force [10]. Non-zero values of frictional forces. were observed at negative normal bending force in Fig. 1, which is caused by the above-mentioned forces.

For simplicity, in the present study, COF was defined as the slope of the friction curve between 0 and $150 \mathrm{nN}$, where the frictional force varies linearly with normal bending force. A similar definition has been employed

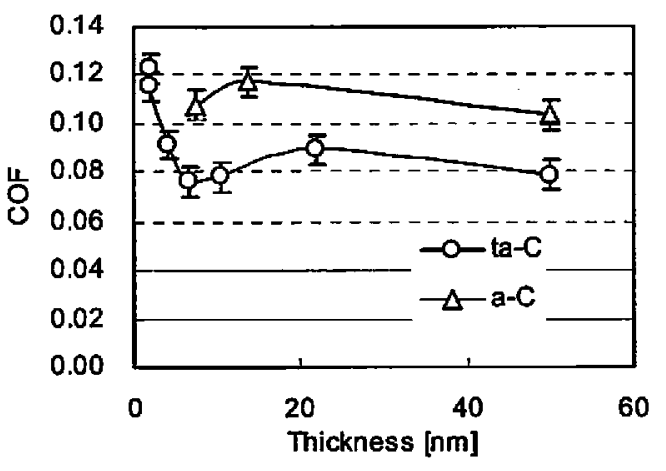

Fig. 2 Coefficient of friction of DLC films as a function of a thickness.

elsewhere [11], and proved to be sufficiently appropriate for the comparison of frictional behavior between different samples. The sum of normal bending force of a cantilever and the adhesion force has been generally used as the normal force. However, in the present experiment, since $\mathrm{COF}$ is obtained by differentiating the friction curves, the absolute value of the normal force is not very important.

As pointed out in Section 2, the reproducibility of FFM measurements is crucial in this study. Before a detailed discussion of the results, we verified the reproducibility of the measured COF. A 50-nm-thick ta-C film was measured a total of seven times, over the course of a series of the measurement. The average value and standard deviation of the $\mathrm{COF}$ were 0.079 and 0.006 , respectively. The scatter in COF was, thus, not so significant, indicating that the tip shape did not change appreciably, and that humidity did not influence the results significantly. Thus the reproducibility of the results was verified. We will next discuss the dependence of COF on thickness.

Figure 2 shows the dependence of COF on film thickness. Measured were ta-C films having a thickness of $1.8,1.9,4,6.5,10.3,22,50 \mathrm{~nm}$ and $\mathrm{a}-\mathrm{C}$ having a thickness of 7.4, $11.5,50 \mathrm{~nm}$. In the case of ta-C films, COF was observed to increase with decreasing thickness, which was in consistent with the results previously reported by Liu [4]. COF of $s p^{2}$ rich a-C films was higher than that of ta-C films.

As a first step toward an understanding of the above observations, we will proceed with a basic theory of friction [10], according to which a frictional force is given by

$$
f_{f}=A \cdot S
$$

where $A$ is the contact area, and $S$ is the shear strength. According to the formula (1), an increase in frictional force should be attributed to an increase of contact area and/or shear strength.

An increase in contact area is classically described by Hertzian theory since a tip of cantilever is spherical. According to this theory, a contact area is expressed as 


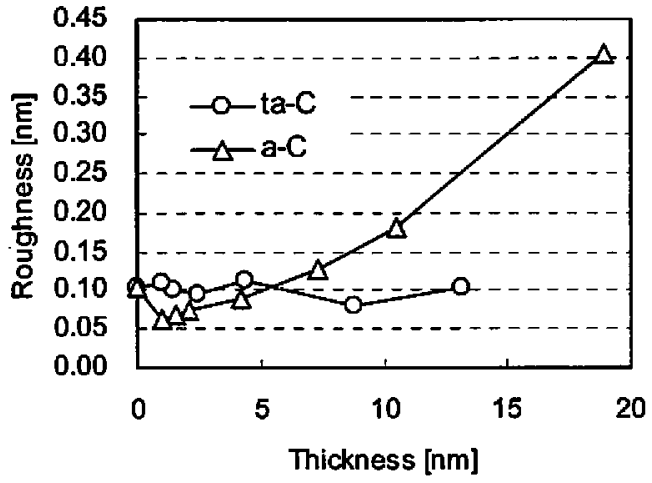

Fig. 3 Roughness of DLC films as a function of a thickness.

$$
A=\pi\left(\frac{R^{*} \cdot f_{n}}{E^{*}}\right)^{2 / 3}
$$

where $R^{*}, E^{*}$, and $f_{n}$ are the relative radius of curvature, effective Young's modulus of the system, and normal force, respectively. The relative radius of curvature is

$$
R^{*}=\frac{R_{1} \cdot R_{2}}{R_{1}+R_{2}}
$$

where $R_{1}$ and $R_{2}$ are the radii of curvature of a tip and sample surface, respectively. When the sample surface is perfectly flat, which means the radius of curvature is infinite, $R^{*}$ is equal to $R_{l}$, i.e., the tip radius. $E^{*}$ is given by

$$
\frac{1}{E^{*}}=\frac{3}{4} \cdot\left(\frac{1-v_{1}{ }^{2}}{E_{1}}+\frac{1-v_{2}{ }^{2}}{E_{2}}\right)
$$

where $v_{i}$ is the Poisson's ratio, and $E_{i}$ is the Young's modulus of the tip and sample surface, respectively; $i=1$, 2.

To discuss the increase in frictional force, we have to pay attentions to both the increase of the contact area and the shear strength. However, it is assumed here that the shear strength does not vary significantly under the condition in this study since it was shown that the surface energies were constant in DLC films prepared by pulse-biasing FCVA, implying insensitivity of surface energy to $s p^{3}$ ratio [12]. We, therefore, focus on the increase in contact area, which will be broken into the decreases in the radius of curvature and effective Young's modulus.

As the relative radius of curvature decreases, the contact area shrinks. In our system, the change in surface roughness could influence the relative radius of curvature. Figure 3 shows the average surface roughness evolution as a function of film thickness. Figure 4 shows the roughest surface among the films evaluated. The surface roughness was obtained from another series of DLC films that were fabricated by the same FCVA and RF magnetron sputtering tools. The roughness of a-C films changed from 0.1 to $0.4 \mathrm{~nm}$ as the thickness changed from 0.05 to $20 \mathrm{~nm}$, while that of ta-C did not change significantly. Thus, the change in the radius of

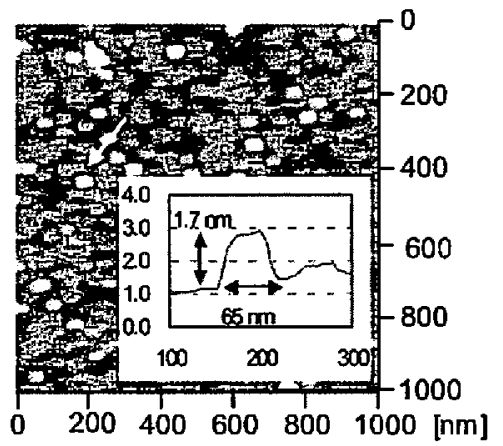

Fig. 4 AFM image of a 50 nm-thick a-C film. The inset shows a cross-sectional view of the curvature indicated by the white arrow.

curvature does not explain the increase of a frictional force of ta-C films because; (i) the significant roughness change was not observed in ta-C films, where the significant increase of COF was observed, and (ii) a change of $R_{2}$ from infinity to $200 \mathrm{~nm}$, which was calculated from the roughest curvature observed in Fig. 4 , corresponds to decrease in COF by about $7 \%$. This is not sufficiently large to explain the results in Fig. 2.

As the effective Young's modulus decreases, a contact area becomes larger. In the case of the contact between the $\mathrm{Si}_{3} \mathrm{~N}_{4}$ tip and DLC on Si substrate, the effective Young's modulus is calculated from the Young's moduli of $\mathrm{Si}_{3} \mathrm{~N}_{4}$ and that of the composite system of DLC on Si substrate. The composite Young's modulus of DLC on Si depends on Young's moduli of $\mathrm{Si}$ and DLC, and the thickness of DLC. When the thickness goes smaller, two mechanisms are possible to reduce the effective Young's modulus, namely (1) the degradation of Young's modulus of ta-C films, and (2) an increasingly dominant role of $\mathrm{Si}$ in a composite Young's modulus. Hereafter, the influences of above two mechanisms are roughly estimated from the simple comparison of $\mathrm{COF}$ among thick ta- $\mathrm{C}$, thin ta-C and bare Si substrate.

Young's modulus of the DLC films is derived from the $s p^{3}$ ratio, since it depends on the rigidity of the individual bonds and the average coordination of the network [1]. According to the research by Ferrari et al., the Young's modulus of DLC films can be expressed by

$$
E=E_{0}\left(Z-Z_{0}\right)^{1.5}=E_{0}\left(x_{s p^{3}}+3-Z_{0}\right)^{1.5}
$$

as a function of $s p^{3}$ ratio, $x_{s p}{ }^{3}$, where E0 is about 483 $\mathrm{GPa}$, and $Z_{0}$ is critical coordination [13]. $Z_{0}$ is computed to be 2.6 in Ferrari's study, which was different from the 2.4 derived from the theory [14]. In this study, we followed the fitting results reported by Ferrari.

To verify the influence of the $s p^{3}$ ratio of DLC films on the increase in contact area, Young's modulus of ta-C and $\mathrm{a}-\mathrm{C}$ films were calculated from the formula (5). Figure 5 shows the $s p^{3}$ ratios of two types of DLC films, which was obtained from XPS measurement and previously reported [5]. At large thickness, the $s p^{3}$ ratios 


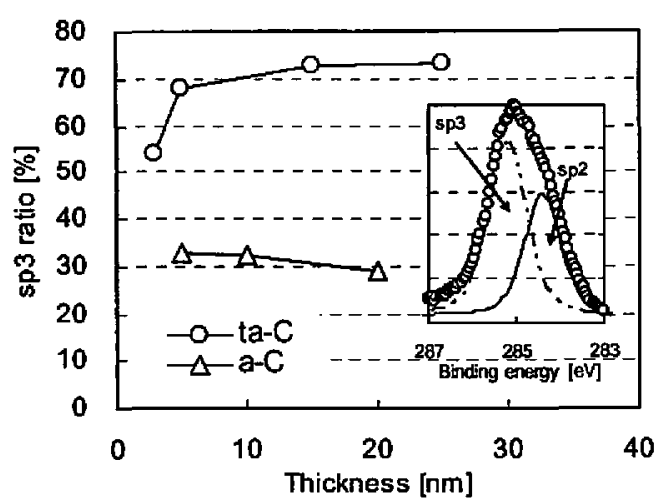

Fig. $5 \mathrm{sp}^{3}$ ratio of DLC films as a function of thickness. The inset shows a typical XPS spectrum.

of ta-C and a-C films were 73 and $30 \%$, respectively. Hence, the Young's moduli of ta-C and a-C films were calculated to be 283 and $596 \mathrm{GPa}$, respectively. The contact area is, then, deprived from the formula (2) and (4), 3.9 and $4.8 \mathrm{~nm}^{2}$ for ta-C and a-C, respectively. The calculation was performed in case of a contact with a $\mathrm{Si}_{3} \mathrm{~N}_{4}$ tip at a normal load of $100 \mathrm{nN}$ and the curvature radius of $20 \mathrm{~nm}$. The difference was quantitatively fairly consistent with $\mathrm{COF}$ results in Fig. 2, where a-C was higher than ta-C by $28 \%$. Eventually, it is suggested that the difference in Young's modulus can explain the difference in COF between ta-C and a-C.

Based on the technique employed above, the two mechanism of modulus reduction are discussed hereafter to verify which one is more significant, (1) the degradation of Young's modulus of ta- $C$ films [5], and (2) a increasingly dominant role of $\mathrm{Si}$ in a composite Young's modulus. As shown in Fig. $5, s p^{3}$ ratio decreased from 73.4 to $54 \%$, as the thickness went down from 25 to $3 \mathrm{~nm}$. The reduction of $s p^{3}$ ratio above corresponds to an increase of contact area by $7 \%$ from the formula (2), (4) and (5). In Fig. 2, an increase of COF by about $16 \%$ was observed for ta-C with decreasing thicknesses. The comparison suggests that it's difficult to explain an increase of COF merely by the transition of $s p^{3}$ to $s p^{2}$ in ta-C films. On the contrary, a Hertzian contact between $\mathrm{Si}$ and $\mathrm{Si}_{3} \mathrm{~N}_{4}$ will give 2 times larger contact area than that of ta-C films, since the Young's modulus of Si substrate is $130 \mathrm{GPa}$ [15]. It is then suggested that COF increase as much as shown in Fig. 2 when the influence of $\mathrm{Si}$ substrate to the composite Young's modulus becomes dominant.

\section{CONCLUSIONS}

To clarify the nano-tribological behavior of ultra-thin DLC films, FFM was performed to measure the COF of two types of DLC films, one type prepared by FCVA and the other by RF magnetron sputtering. The measurements were conducted under atmospheric conditions, and at a load that did not cause fracture. The results showed an increase in the $\mathrm{COF}$ of ta-C films at the thickness below $10 \mathrm{~nm}$. The increase of COF can be explained by the increase of a contact area due to the reduction of a composite Young's modulus of DLC on Si substrate. Quantitative comparison suggested that a role of Si substrate becomes increasingly significant in the composite Young's modulus with decreasing thickness, which leads to a significant reduction of effective Young's modulus. It is thus suggested that at the thickness below $10 \mathrm{~nm}$, there is a necessity to design the nano-tribological system with a special attention on a substrate material.

\section{REFERENCES}

[1] J. Robertson, Materials Science and Engineering, R37, 129 (2002)

[2] S. Sundararajan, and B. Bhushan, Wear 225-229, 678 (1999)

[3] X.-G. Ma, K. Komvopoulos, D. Wan, D.B. Bogy, and Y.-S. Kim, Wear, 254, 1010 (2003)

[4] D. Liu, G. Benstetter, and E. Lodermeier, Surf. and Coat. Technol,, 188-189, 511 (2004)

[5] N. Yasui, H. Inaba, and S. Sasaki,, e-J. Surf. Sci. Nanotech. 4, 1 (2006)

[6] H. Inaba, K. Furusawa, S. Hirano, S. Sasaki, S. Todoroki, M. Yamasaka, and M. Endou, Jpn. J. Appl. Phys., 42, 2824 (2003)

[7] R. Lossy, A. L. Pappas, R. A. Roy, J. P. Doyle, and J. Bruley, J. Appl. Phys., 77(9), 4750 (1995)

[8] A. Khan, J. Philip, and P. Hess, J. Appl. Phys., 95, 1667 (2004)

[9] J. Hu, X.-d. Xiao, D. F. Ogletree, and M. Salmeron, Surf. Sci. 327, 358 (1995)

[10] U. D. Schwar, O. Zwomer, P. Koster, and R. Wiesendanger, Phys. Rev. B, 56, 6987 (1997)

[11] E. Liu, B. Blanpain, J.-P. Celis, and J.R. Roos, J. Appl. Phys., 84, 4859 (1998)

[12] J. S. Chen, S. P. Lau, B.K. Tay, G. Y. Chen, Z. Sun, Y. Y. Tan, G. Tan, and J. W. Chai, J. Appl. Phys., 89, $7814(2001)$

[13] A. C. Ferrari, J. Robertson, M. G. Beghi, C. E. Bottani, R. Ferulano, and R. Pastorelli, Appl. Phys. Lett., 75, 1893 (1999)

[14] H. He, and M. E. Thorpe, Phys. Rev. Lett. 54, 2107 (1985)

[15] D. R. Franca and A. Blouin, Meas. Sci. Technol. 15 859 (2004)

(Received December 9, 2006;Accepted February 8, 2007) 